\title{
SOME PROPERTIES OF THE ZERO-DIVISOR GRAPH FOR THE RING OF GAUSSIAN INTEGERS MODULO $n$
}

\author{
EMAD ABU OSBA \\ Department of Mathematics, Faculty of Science, University of Jordan, \\ Amman 11942, Jordan \\ e-mail:eabuosba@ju.edu.jo \\ SALAH AL-ADDASI \\ Department of Mathematics, Faculty of Science, Hashemite University, \\ Zarqa13115, Jordan \\ e-mail:salah@hu.edu.jo \\ and BASEM AL-KHAMAISEH \\ Department of Mathematics, Faculty of Science, University of Jordan, \\ Amman 11942, Jordan \\ e-mail:basem198426@yahoo.com
}

(Received 14 May 2009; revised 28 February 2010; accepted 24 September 2010)

\begin{abstract}
This paper is a continuation for the study of the zero-divisor graph for the ring of Gaussian integers modulo $\mathrm{n}, \Gamma\left(\mathbb{Z}_{n}[i]\right)$ in $[8]($ Emad Abu Osba, Salah Al-Addasi and Nafez Abu Jaradeh. Zero divisor graph for the ring of Gaussin integers modulo n. Comm. Algebra 36(10) (2008), 3865-3877). It is investigated, when is $\Gamma\left(\mathbb{Z}_{n}[i]\right)$ locally $\mathrm{H}$, Hamiltonian or bipartite graph? A full characterisation for the chromatic number and the radius is also given.
\end{abstract}

2010 Mathematics Subject Classification. 13A99, 05C15

1. Introduction. Let $R$ be a commutative ring, $Z(R)$ the set of zero divisors of $R$, and $Z^{*}(R)=Z(R)-\{0\}$. The zero-divisor graph of $R, \Gamma\left(Z^{*}(R)\right)$, usually written as $\Gamma(R)$, is the simple graph in which each element of $Z^{*}(R)$ is a vertex, i.e. $V(\Gamma(R))=$ $Z^{*}(R)$, and two distinct vertices $x$ and $y$ are adjacent if and only if $x y=0$. For more details about the zero-divisor graph of a ring, the reader may refer to [1].

The set of all complex numbers $a+i b$, where $a$ and $b$ are integers, forms a Euclidean domain with the usual complex number operations and Euclidean norm $|a+i b|=$ $a^{2}+b^{2}$. This domain is denoted by $\mathbb{Z}[i]$ and is called the ring of Gaussian integers. It is clear that $a+i b$ is a unit in $\mathbb{Z}[i]$ if and only if $|a+i b|=1$, which implies that the only units in $\mathbb{Z}[i]$ are $1,-1, i$ and $-i$.

Let $n$ be a natural number and let $\langle n\rangle$ be the principal ideal generated by $n$ in $\mathbb{Z}[i]$. Then the factor ring $\mathbb{Z}[i] /<n>$ is isomorphic to $\mathbb{Z}_{n}[i]=\left\{\bar{a}+i \bar{b}: \bar{a}, \bar{b} \in \mathbb{Z}_{n}\right\}$, which implies that $\mathbb{Z}_{n}[i]$ is a principal ideal ring. The ring $\mathbb{Z}_{n}[i]$ is called the ring of Gaussian integers modulo $\mathrm{n}$.

Let $2=-i(1+i)^{2}$, so $\mathbb{Z}_{2^{m}}[i]$ is isomorphic to the local $\operatorname{ring} \mathbb{Z}[i] /<(1+i)^{2 m}>$ with only maximal ideal $<\overline{1}+i>$. If $q$ is a prime integer such that $q \equiv 3(\bmod 4)$, then $\mathbb{Z}_{q}[i]$ is a field, while for $m>1, \mathbb{Z}_{q^{m}}[i]$ is a local ring, which is not a field with 
maximal ideal $<\bar{q}>$. If $p$ is a prime integer such that $p \equiv 1(\bmod 4)$, then there exists $a, b \in \mathbb{N}$ such that $p=a^{2}+b^{2}=(a+i b)(a-i b)$, both factors are Gaussian primes and $\mathbb{Z}_{p^{m}}[i] \simeq\left(\mathbb{Z}[i] /<(a+i b)^{m}>\right) \times\left(\mathbb{Z}[i] /<(a-i b)^{m}>\right) \simeq \mathbb{Z}_{p^{m}} \times \mathbb{Z}_{p^{m}}$, see $[\mathbf{5}$, Theorem 5]. In this paper, the integers $q$ and $q_{j}$ are used implicitly to denote prime integers congruent to 3 modulo 4 , while $p$ and $p_{s}$ likewise denote prime integers congruent to 1 modulo 4.

A complete graph with $n$ vertices is denoted by $K_{n}$. A complete bipartite graph with partite sets having $n$ and $m$ vertices is denoted by $K_{n, m}$. The edgeless graph with $n$ vertices is denoted by $n K_{1}$.

In $[8]$, the authors find the diameter and girth of $\Gamma\left(\mathbb{Z}_{n}[i]\right)$. They investigated, when is $\Gamma\left(\mathbb{Z}_{n}[i]\right)$ complete, complete bipartite, regular, planar or Eulerian?

This paper is a continuation of the work done in $[\mathbf{8}]$. We will study, when is $\Gamma\left(\mathbb{Z}_{n}[i]\right)$ locally $\mathrm{H}$, Hamiltonian or bipartite? We will find the radius and the chromatic number in terms of $n$.

For any undefined terms, the reader may contact [8] and [2].

2. When is $\Gamma\left(\mathbb{Z}_{n}[i]\right)$ locally $\mathbf{H}$ ?. A graph in which all vertices have the same degree is called a regular graph. If all vertices in a graph $G$ have neighbourhoods that are isomorphic to the same graph $H$, then $G$ is said to be locally $H$, see [3]. A graph $G$ of diameter $d$ is called distance regular with parameters $\left\{p_{i, j}^{k}: 0 \leq i, j, k \leq d\right\}$ if for each triple $(i, j, k)$ and for any pair $(u, v)$ of vertices of $G$ such that $d(u, v)=k$, the number of vertices at distance $i$ from $u$ and distance $j$ from $v$ is $p_{i, j}^{k}$, each of these numbers $p_{i, j}^{k}$ is independent of the particular choice of vertices. A special class of distance regular graphs is that of strongly regular graphs. A graph $G$ is called strongly regular if it is distance regular of diameter 2, see [6].

In this section we investigate the cases in which the graph $\Gamma\left(\mathbb{Z}_{n}[i]\right)$ is locally $H$.

THEOREM 1. The graph $\Gamma\left(\mathbb{Z}_{n}[i]\right)$ is locally $H$ if and only if $n=2$ or $n=p$ or $n=q^{2}$.

Proof. The graph $\Gamma\left(\mathbb{Z}_{2}[i]\right)$ contains only one vertex; namely $\overline{1}+i$ and so $\Gamma\left(\mathbb{Z}_{2}[i]\right)$ is locally $\phi$.

If $n=p$, then $n=a^{2}+b^{2}$ for some $a, b \in \mathbb{N}$, and the vertex set of $\Gamma\left(\mathbb{Z}_{n}[i]\right)$ is $(\langle\bar{a}+i \bar{b}\rangle \cup\langle\bar{a}-i \bar{b}\rangle)-\{\overline{0}\}$. In this case, $\Gamma\left(\mathbb{Z}_{n}[i]\right)$ is the complete bipartite graph $K_{n-1, n-1}$. Hence the graph $\Gamma\left(\mathbb{Z}_{n}[i]\right)$ is locally $(n-1) K_{1}$.

If $n=q^{2}$, then the vertex set of $\Gamma\left(\mathbb{Z}_{n}[i]\right)$ is $\langle\bar{q}\rangle-\{\overline{0}\}$. In this case, $\Gamma\left(\mathbb{Z}_{n}[i]\right)$ is the complete graph $K_{n-1}$. Hence, the graph $\Gamma\left(\mathbb{Z}_{n}[i]\right)$ is locally $K_{n-2}$.

It was shown in $[8]$ that the graph $\Gamma\left(\mathbb{Z}_{n}[i]\right)$ is regular if and only if $n=2$ or $n=p$ or $n=q^{2}$. Hence $\Gamma\left(\mathbb{Z}_{n}[i]\right)$ cannot be locally $\mathrm{H}$ for any other case.

Since the regular complete bipartite graph $K_{n, n}, n \geq 2$ is strongly regular and the complete graph $K_{n}$ is distance regular, one can deduce the following corollary.

COROLlARY 2. (a)The graph $\Gamma\left(\mathbb{Z}_{n}[i]\right)$ is locally $H$ if and only if it is distance regular if and only if it is regular.

(b) The graph $\Gamma\left(\mathbb{Z}_{n}[i]\right)$ is strongly regular if and only if $n=p$.

3. When is $\Gamma\left(\mathbb{Z}_{n}[i]\right)$ Hamiltonian?. A component of an undirected graph is a subgraph in which any two vertices are connected to each other by paths, and to which no more vertices or edges can be added while preserving its connectivity, that is, it is a maximal connected subgraph. For a graph $G$, let $\mathbf{c}(\mathbf{G})$ denote the number of 
components. A Hamiltonian cycle of a graph $G$ is a cycle that contains every vertex of $G$. A graph is Hamiltonian if it contains a Hamiltonian cycle.

The name 'Hamiltonian cycle' arises from the fact that Sir William Hamilton investigated their existence in the dodecahedron graph. One of the major unsolved problems of graph theory is to obtain simple characterisations for Hamiltonian graphs. Most existing theorems have the form, 'if $G$ has enough edges, then $G$ is Hamiltonian'. Probably, the most celebrated of these is the following result because of G. A. Dirac, see [10].

Proposition 3. If $G$ is a graph with $n(\geq 3)$ vertices, and if $\operatorname{deg}(v) \geq \frac{n}{2}$ for each vertex $v$, then $G$ is Hamiltonian.

Another well-known existence theorem on Hamiltonian graphs is the following, see, for example, [10, p. 38].

Proposition 4. If $G$ is a Hamiltonian graph and $S$ is any non-empty proper subset of vertices in $G$, then $c(G-S) \leq|S|$.

We will use these two propositions to characterise when the graph $\Gamma\left(\mathbb{Z}_{n}[i]\right)$ is Hamiltonian. We will show that $\Gamma\left(\mathbb{Z}_{n}[i]\right)$ is Hamiltonian if and only if $n=p$ or $n=q^{2}$.

THEOREM 5. For each $m \geq 1$, the graph $\Gamma\left(\mathbb{Z}_{2^{m}}[i]\right)$ is not Hamiltonian.

Proof. The graph $\Gamma\left(\mathbb{Z}_{2}[i]\right)$ is the trivial graph $K_{1}$ which is not Hamiltonian. For $m>1$, the vertex set $V\left(\Gamma\left(\mathbb{Z}_{2^{m}}[i]\right)\right)=\langle\overline{1}+i\rangle-\{\overline{0}\}$ and in this graph $(\overline{1}+i)(\overline{1}-i)=\overline{2} \neq$ $\overline{0}$ and all vertices are adjacent to $(\overline{1}+i)^{2 m-1}$. Also $\operatorname{deg}(\overline{1}+i)=1=\operatorname{deg}(\overline{1}-i)$, see [8] . Let $S=\left\{(\overline{1}+i)^{2 m-1}\right\}$ and let $H=\{\overline{1}+i, \overline{1}-i\}$. Then $c\left(\Gamma\left(\mathbb{Z}_{2^{m}}[i]\right)-S\right) \geq|H|=2>$ $1=|S|$. So it follows by Proposition 4 that $\Gamma\left(\mathbb{Z}_{2^{m}}[i]\right)$ is not Hamiltonian.

THEOREM 6. The graph $\Gamma\left(\mathbb{Z}_{p^{m}}[i]\right)$ is Hamiltonian if and only if $m=1$.

Proof. Let $p=a^{2}+b^{2}$ for some $a, b \in \mathbb{N} . \Gamma\left(\mathbb{Z}_{p}[i]\right)$ is the complete bipartite graph $K_{p-1, p-1}$ with the two vertex sets $V_{1}=\langle\bar{a}+i \bar{b}\rangle-\{\overline{0}\}$ and $V_{2}=\langle\bar{a}-i \bar{b}\rangle-\{\overline{0}\}$. So it is clear that $\Gamma\left(\mathbb{Z}_{p}[i]\right)$ is a Hamiltonian graph. Now let $m>1, \mathbb{Z}_{p^{m}}[i] \simeq \mathbb{Z}_{p^{m}} \times$ $\mathbb{Z}_{p^{m}}$, so let $S=\left\{\left(\overline{0}, \alpha p^{m-1}\right) \in \mathbb{Z}_{p^{m}} \times \mathbb{Z}_{p^{m}}: \operatorname{gcd}(\alpha, p)=1\right\}, H_{1}=\left\{(\overline{1}, \alpha p) \in \mathbb{Z}_{p^{m}} \times \mathbb{Z}_{p^{m}}:\right.$ $\operatorname{gcd}(\alpha, p)=1\}$ and $H_{2}=\left\{(\overline{2}, \alpha p) \in \mathbb{Z}_{p^{m}} \times \mathbb{Z}_{p^{m}}: \operatorname{gcd}(\alpha, p)=1\right\}$. Then $\left|H_{1}\right|=\left|H_{2}\right| \geq$ $p-1=|S|$. Elements of $H_{1}$ and $H_{2}$ are adjacent only to elements of $S$. Then $c\left(\Gamma\left(\mathbb{Z}_{p^{m}} \times \mathbb{Z}_{p^{m}}\right)-S\right) \geq\left|H_{1}\right|+\left|H_{2}\right|>|S|$. Hence $\Gamma\left(\mathbb{Z}_{p^{m}}[i]\right)$ is not Hamiltonian.

LemMA 7. Let $m>1$ and let $\alpha, \beta \in\{0, q, 2 q, 3 q, \ldots,(q-1) q\} \subseteq \mathbb{Z}_{q^{m}}[i]$ such that $(\alpha, \beta) \neq(0,0)$. Then the set $\{\bar{x}+i \bar{y}:(\bar{x}+i \bar{y})(\bar{\alpha}+i \bar{\beta})=\overline{0}, \bar{x}+i \bar{y} \neq \overline{0}\}=\left\langle\bar{q}^{m-1}\right\rangle-\{\overline{0}\}$.

Proof. Assume that $(\overline{a q}+\overline{b q} i)(\bar{x}+i \bar{y})=\overline{0}$, where $a, b \in\{0,1,2, \ldots, q-1\}$ but not both are zeroes. Then we have:

$a x-b y=q^{m-1} l_{1}$,

$b x+a y=q^{m-1} l_{2}$.

So $\left(a^{2}+b^{2}\right) x=q^{m-1}\left(a l_{1}+b l_{2}\right)$ and $\left(a^{2}+b^{2}\right) y=q^{m-1}\left(a l_{2}-b l_{1}\right)$, which implies that $q^{m-1} \mid x$ and $q^{m-1} \mid y$, because if $q \mid\left(a^{2}+b^{2}\right)$, then $\left(a^{-1} b\right)^{2} \equiv-1(\bmod q)$ which contradicts the fact that $q \equiv 3(\bmod 4)$. Thus $\bar{x}+i \bar{y} \in\left\langle\bar{q}^{m-1}\right\rangle-\{\overline{0}\}$.

THEOREM 8. The graph $\Gamma\left(\mathbb{Z}_{q^{m}}[i]\right)$ is Hamiltonian if and only if $m=2$.

Proof. $\mathbb{Z}_{q}[i]$ is a filed and so $\Gamma\left(\mathbb{Z}_{q}[i]\right)$ is the empty graph. $\Gamma\left(\mathbb{Z}_{q^{2}}[i]\right)$ is the complete graph $K_{q^{2}-1}$, see [8], which is a Hamiltonian graph. Now let $m>2$. Then the 
vertex set of $\Gamma\left(\mathbb{Z}_{q^{m}}[i]\right)$ is $\langle\bar{q}\rangle-\{\overline{0}\}$. Let $S=\left\langle\bar{q}^{m-1}\right\rangle-\{\overline{0}\}$ and let $H=\{\bar{\alpha}+i \bar{\beta}: \alpha, \beta \in$ $\{0, q, 2 q, 3 q, \ldots,(q-1) q\},(\alpha, \beta) \neq(0,0)\}$. Then $H \subseteq V\left(\Gamma\left(\mathbb{Z}_{q^{m}}[i]\right)\right)-S$, and it follows by Lemma 7 that $c\left(\Gamma\left(\mathbb{Z}_{q^{m}}[i]\right)-S\right)>|H|=q^{2}-1=|S|$. So, it follows by Proposition 4 that $\Gamma\left(\mathbb{Z}_{q^{m}}[i]\right)$ is not Hamiltonian.

LEMMA 9. If $R=R_{1} \times R_{2}$ with $\left|\operatorname{reg}\left(R_{1}\right)\right|>1$ and $\left|Z^{*}\left(R_{2}\right)\right|>1$, then $\Gamma(R)$ is not Hamiltonian.

Proof. Let $S=\left\{(0, v): v \in Z^{*}\left(R_{2}\right)\right\}$ and let $H=\left\{(u, v): u \in \operatorname{reg}\left(R_{1}\right)\right.$ and $v \in$ $\left.Z^{*}\left(R_{2}\right)\right\}$. Then the elements of $H$ are adjacent only to elements of $S$ and $c(\Gamma(R)-S) \geq|H|=\left|\operatorname{reg}\left(R_{1}\right)\right| \times\left|Z^{*}\left(R_{2}\right)\right| \geq 2\left|Z^{*}\left(R_{2}\right)\right|>\left|Z^{*}\left(R_{2}\right)\right|=|S|$. Thus $\Gamma(R)$ is not Hamiltonian.

THEOREM 10. If an integer $n$ is divisible by at least two distinct primes, then $\Gamma\left(\mathbb{Z}_{n}[i]\right)$ is not Hamiltonian.

Proof. If $n=2 t$ with $\operatorname{gcd}(2, t)=1$, then $\mathbb{Z}_{n}[i] \simeq \mathbb{Z}_{2}[i] \times \mathbb{Z}_{t}[i]$. Take $S=\{(\overline{1}+i, \overline{0})\}$ and $H=\left\{(\overline{1}+i, v): v \in U\left(\mathbb{Z}_{t}[i]\right)\right\}$. Then the vertices of $H$ are adjacent only to $(\overline{1}+i, \overline{0})$ and hence $c\left(\Gamma\left(\mathbb{Z}_{2}[i] \times \mathbb{Z}_{t}[i]\right)-S\right) \geq|H|>1=|S|$, so $\Gamma\left(\mathbb{Z}_{2 t}[i]\right)$ is not Hamiltonian. For the other cases, if $n=m k$ with $m, k>2$ and $\operatorname{gcd}(m, k)=1$, then $\mathbb{Z}_{n}[i] \simeq \mathbb{Z}_{m}[i] \times \mathbb{Z}_{k}[i]$. If neither $\mathbb{Z}_{m}[i]$ nor $\mathbb{Z}_{k}[i]$ is a field, then the result follows immediately from Lemma 9. So assume that both $\mathbb{Z}_{m}[i]$ and $\mathbb{Z}_{k}[i]$ are fields with $m<k$. Let $H=\{(\overline{0}, v): v \in$ $\left.\left(\mathbb{Z}_{k}[i]\right)^{*}\right\}$ and let $S=\left\{(u, \overline{0}): u \in\left(\mathbb{Z}_{m}[i]\right)^{*}\right\}$. Then elements of $H$ are adjacent only to elements of $S$ and $c\left(\Gamma\left(\mathbb{Z}_{n}[i]\right)-S\right)=|H|=k^{2}-1>m^{2}-1=|S|$. Thus $\Gamma\left(\mathbb{Z}_{n}[i]\right)$ is not Hamiltonian.

Combining those results on Hamiltonian graphs together with Theorem 1 and Corollary 2, we can get:

COROLlary 11. For $n>2$, the following are equivalent:

(1) $\Gamma\left(\mathbb{Z}_{n}[i]\right)$ is Hamiltonian.

(2) $\Gamma\left(\mathbb{Z}_{n}[i]\right)$ is locally $H$.

(3) $\Gamma\left(\mathbb{Z}_{n}[i]\right)$ is regular.

(4) $\Gamma\left(\mathbb{Z}_{n}[i]\right)$ is distance regular.

(5) $n=p$ or $n=q^{2}$.

4. The radius of $\Gamma\left(\mathbb{Z}_{n}[i]\right)$. The eccentricity of a vertex $v$ of a connected graph $G$ is the distance between $v$ and a vertex farthest from $v$. The minimum eccentricity among the vertices of $G$ is its radius, which is denoted by $\operatorname{rad}(G)$.

Abu Osba et al. have shown in [8, Theorem 19] that the domination number of $\Gamma\left(\mathbb{Z}_{n}[i]\right)$ is 1 if and only if $n=q^{m}$, where $m>1$ or $n=2^{m}$, which implies the following result, since a vertex in a dominating set of cardinality 1 has the minimum eccentricity.

TheOREM 12. For any integer $n>1$.

(1) $\operatorname{rad}\left(\Gamma\left(\mathbb{Z}_{n}[i]\right)\right)=0$ if and only if $n=2$.

(2) $\operatorname{rad}\left(\Gamma\left(\mathbb{Z}_{n}[i]\right)\right)=1$ if and only if $n=2^{m}$ or $q^{m}$, where $m>1$.

It was shown in Anderson and Livingston [1, 2.3] that for a commutative ring $R$, the graph $\Gamma(R)$ is connected and has diameter at most 3. Therefore, in view of Theorem 12 , if $n \neq 2^{m}$ or $q^{m}$, then $\operatorname{rad}\left(\Gamma\left(\mathbb{Z}_{n}[i]\right)\right) \in\{2,3\}$. Now we consider the case that $n=p^{m}$.

THEOREM 13. For any integer $m \geq 1, \operatorname{rad}\left(\Gamma\left(\mathbb{Z}_{p^{m}}[i]\right)\right)=2$. 
Proof. Let $p=a^{2}+b^{2}$. As shown in $\left[\mathbf{8}\right.$, Theorem 20], the set $\left\{(\bar{a}+i \bar{b})^{m}(\bar{a}-\right.$ $\left.i \bar{b})^{m-1},(\bar{a}+i \bar{b})^{m-1}(\bar{a}-i \bar{b})^{m}\right\}$ is a minimum dominating set of $\Gamma\left(\mathbb{Z}_{p^{m}}[i]\right)$. Thus $\operatorname{rad}\left(\Gamma\left(\mathbb{Z}_{p^{m}}[i]\right)\right)>1$. Since $(\bar{a}+i \bar{b})^{m}(\bar{a}-i \bar{b})^{m-1}$ is adjacent to $(\bar{a}+i \bar{b})^{m-1}(\bar{a}-i \bar{b})^{m}$, we have for any vertex $\alpha$ of $\Gamma\left(\mathbb{Z}_{p^{m}}[i]\right)$ which is not adjacent to $(\bar{a}+i \bar{b})^{m}(\bar{a}-i \bar{b})^{m-1}$, the vertex $(\bar{a}+i \bar{b})^{m-1}(\bar{a}-i \bar{b})^{m}$ is a common neighbour of $(\bar{a}+i \bar{b})^{m}(\bar{a}-i \bar{b})^{m-1}$ and $\alpha$. Therefore, the vertex $(\bar{a}+i \bar{b})^{m}(\bar{a}-i \bar{b})^{m-1}$ has eccentricity 2 , and hence $\operatorname{rad}\left(\Gamma\left(\mathbb{Z}_{p^{m}}[i]\right)\right)=2$.

The following result determines the radius for the remaining case in which $n$ has at least two distinct prime factors.

THEOREM 14. Let $n$ be a positive integer with at least two distinct prime factors. Then $\operatorname{rad}\left(\Gamma\left(\mathbb{Z}_{n}[i]\right)\right)=2$.

Proof. Let $n=t^{m} k$, where $t$ is a prime integer and $\operatorname{gcd}(t, k)=1$. By Theorem 12, $\operatorname{rad}\left(\Gamma\left(\mathbb{Z}_{n}[i]\right)\right)>1$. So it would be enough to find a vertex in $\Gamma\left(\mathbb{Z}_{n}[i]\right)$ with eccentricity 2. We have $\Gamma\left(\mathbb{Z}_{n}[i]\right) \simeq \Gamma\left(\mathbb{Z}_{t^{m}}[i] \times \mathbb{Z}_{k}[i]\right)$. Note that the vertex set of $\Gamma\left(\mathbb{Z}_{t^{m}}[i] \times \mathbb{Z}_{k}[i]\right)$ is $A_{1} \cup A_{2} \cup A_{3} \cup A_{4}$, where

$$
\begin{aligned}
& A_{1}=\left\{(x, \overline{0}): x \in \mathbb{Z}_{t^{m}}[i]-\{\overline{0}\}\right\}, \\
& A_{2}=\left\{(\overline{0}, y): y \in \mathbb{Z}_{k}[i]-\{\overline{0}\}\right\}, \\
& A_{3}=\left\{(x, z): x \in \mathbb{Z}_{t^{m}}[i]-\{\overline{0}\}, z \in Z^{*}\left(\mathbb{Z}_{k}[i]\right)\right\} \text { and } \\
& A_{4}=\left\{(z, y): z \in Z^{*}\left(\mathbb{Z}_{t^{m}}[i]\right), y \in \mathbb{Z}_{k}[i]-\{\overline{0}\}\right\},
\end{aligned}
$$

where $A_{3}$ is empty when $k=q_{1}$ for some $q_{1}$, and $A_{4}$ is empty when $t^{m}=q_{2}$ for some $q_{2}$. Consider the vertex $v=(a, \overline{0})$, where $a$ is a vertex of $\Gamma\left(\mathbb{Z}_{t^{m}}[i]\right)$ with minimum eccentricity. We will show that $v$ has eccentricity 2 in $\Gamma\left(\mathbb{Z}_{t^{m}}[i] \times \mathbb{Z}_{k}[i]\right)$. Since every vertex in $A_{1}$ is adjacent to every vertex in $A_{2}$, we have $d(v, \alpha) \leq 2$ for every $\alpha \in A_{1} \cup A_{2}$. If $(x, z) \in A_{3}$, then there exists an element $z_{1} \in Z^{*}\left(\mathbb{Z}_{k}[i]\right)$ such that $z z_{1}=\overline{0}$, and hence $\left(\overline{0}, z_{1}\right)$ is a common neighbour of $(a, \overline{0})$ and $(x, z)$. Thus $d(v,(x, z)) \leq 2$. Finally, if $(z, y) \in A_{4}$, then by the choice of $a$ and according to Theorem 12 or Theorem 13, we have $d(a, z) \leq 2$. Then either $z=a$ or $a_{-} z \in E\left(\Gamma\left(\mathbb{Z}_{t^{m}}[i]\right)\right)$ or $a$ and $z$ have a common neighbour $z_{1}$ in $\Gamma\left(\mathbb{Z}_{t^{m}}[i]\right)$. Therefore, if $z \neq a$, then either $(a, \overline{0})$ is adjacent to $(z, y)$, or the vertex $\left(z_{1}, \overline{0}\right)$ is a common neighbour of $(a, \overline{0})$ and $(z, y)$, and hence in any case we have $d(v,(z, y)) \leq 2$. So suppose that $z=a$. Now if $t^{m}=2$, then $a=\overline{1}+\overline{1} i=z$ and $(a, \overline{0})$ is adjacent to $(z, y)$, which implies that $d(v,(z, y))=1$. If $t^{m} \neq 2$, then $a$ has a neighbour $x_{1}$ in $\Gamma\left(\mathbb{Z}_{t^{m}}[i]\right)$, and hence $\left(x_{1}, \overline{0}\right)$ is a common neighbour of $(a, \overline{0})$ and $(z, y)$, which implies that $d(v,(z, y)) \leq 2$. Therefore, the vertex $v$ has eccentricity at most 2 and hence its eccentricity is 2 . Thus $\operatorname{rad}\left(\Gamma\left(\mathbb{Z}_{n}[i]\right)\right)=2$.

Summarising the results in the three theorems of this section, we have: for any integers $n>1, m>1$ with $n \neq q$ for any $q$,

$$
\operatorname{rad}\left(\Gamma\left(\mathbb{Z}_{n}[i]\right)\right)= \begin{cases}0 & n=2 \\ 1 & n=2^{m} \text { or } q^{m} . \\ 2 & \text { otherwise }\end{cases}
$$

5. When is $\Gamma\left(\mathbb{Z}_{n}[i]\right)$ bipartite?. A subset $S$ of vertices of a graph $G$ is called independent if no pair of vertices of $S$ are adjacent.

Abu Osba et al. have shown in $\left[\mathbf{8}\right.$, Theorem 17] that $\Gamma\left(\mathbb{Z}_{n}[i]\right)$ is complete bipartite if and only if $n=p$ or $n=q_{1} q_{2}$. In this section, we will determine all values of $n$ for 
which $\Gamma\left(\mathbb{Z}_{n}[i]\right)$ is bipartite. We start by proving that $\Gamma\left(\mathbb{Z}_{2 q}[i]\right)$ is a sequential join of four graphs. The sequential join $G_{1}+G_{2}+\cdots+G_{k}$ of the graphs $G_{1}, G_{2}, \cdots, G_{k}$ is the graph formed by taking one copy of each of the graphs $G_{1}, G_{2}, \cdots, G_{k}$ and adding in additional edges from each vertex of $G_{j}$ to each vertex of $G_{j+1}$, for $j=1,2, \ldots, k-1$, see [2].

LEMMA 15. The graph $\Gamma\left(\mathbb{Z}_{2 q}[i]\right)$ is isomorphic to the sequential join $\left(q^{2}-1\right) K_{1}+$ $K_{1}+\left(q^{2}-1\right) K_{1}+2 K_{1}$.

Proof. The graph $\Gamma\left(\mathbb{Z}_{2 q}[i]\right)$ is isomorphic to $\Gamma\left(\mathbb{Z}_{2}[i] \times \mathbb{Z}_{q}[i]\right)$. The vertex set of $\Gamma\left(\mathbb{Z}_{2}[i] \times \mathbb{Z}_{q}[i]\right)$ is $A_{1} \cup A_{2} \cup A_{3}$, where

$A_{1}=\left\{(x, \overline{0}): x \in \mathbb{Z}_{2}[i]-\{\overline{0}\}\right\}$,

$A_{2}=\left\{(\overline{0}, y): y \in \mathbb{Z}_{q}[i]-\{\overline{0}\}\right\}$ and

$A_{3}=\left\{(\overline{1}+\overline{1} i, y): y \in \mathbb{Z}_{q}[i]-\{\overline{0}\}\right\}$.

Then the set of vertices adjacent to $(\overline{1}+\overline{1} i, \overline{0})$ is $A_{2} \cup A_{3}$. Since $\mathbb{Z}_{q}[i]$ is a field, we have $A_{2} \cup A_{3}$ as an independent set of vertices. Obviously $A_{1}$ is also an independent set of vertices. Therefore, since the set of vertices adjacent to $(\overline{1}, \overline{0})$ which is equal to $A_{2}$ which equals the set of vertices adjacent to $(i, \overline{0})$, we have $\Gamma\left(\mathbb{Z}_{2}[i] \times \mathbb{Z}_{q}[i]\right)=G_{1}+$ $G_{2}+G_{3}+G_{4}$, where $G_{1}, G_{2}, G_{3}, G_{4}$ are the graphs induced by $A_{3},\{(\overline{1}+\overline{1} i, \overline{0})\}, A_{2}$ and $\{(\overline{1}, \overline{0}),(i, \overline{0})\}$, respectively.

Since each of the sets $A_{2}$ and $A_{3}$ in the proof of Lemma 15 has cardinality $q^{2}-1$, we have the following result.

COROLlARY 16. The graph $\Gamma\left(\mathbb{Z}_{2 q}[i]\right)$ is bipartite with partite sets of cardinalities 3 and $2\left(q^{2}-1\right)$.

Now we are in a position to determine precisely when $\Gamma\left(\mathbb{Z}_{n}[i]\right)$ is bipartite. Note that $\Gamma\left(\mathbb{Z}_{2}[i]\right)$ is the trivial graph $K_{1}$.

THEOREM 17. For any integer $n>2$, the following are equivalent:

(1) $\Gamma\left(\mathbb{Z}_{n}[i]\right)$ is bipartite.

(2) $\Gamma\left(\mathbb{Z}_{n}[i]\right)$ is triangle free.

(3) $n=p$ or $2 q$ or $q_{1} q_{2}$.

Proof. (1) $\Rightarrow$ (2) Any bipartite graph has no triangle.

$(2) \Rightarrow(3)$ Let $\Gamma\left(\mathbb{Z}_{n}[i]\right)$ be triangle free. Since $\mathbb{Z}_{q}[i]$ is a field, by $[\mathbf{8}$, Theorem 14$]$, we have $n=p$ or $2 q$ or $q_{1} q_{2}$.

(3) $\Rightarrow$ (1) Let $n=p$ or $2 q$ or $q_{1} q_{2}$. Then, by [8, Theorem 17] and Corollary 16, $\Gamma\left(\mathbb{Z}_{n}[i]\right)$ is bipartite.

Since $\Gamma\left(\mathbb{Z}_{p}[i]\right) \simeq K_{p-1, p-1}, \Gamma\left(\mathbb{Z}_{q_{1} q_{2}}[i]\right) \simeq K_{q_{1}-1, q_{2}-1}$, see $[\mathbf{8}]$, and $\Gamma\left(\mathbb{Z}_{2 q}[i]\right) \simeq\left(q^{2}-\right.$ 1) $K_{1}+K_{1}+\left(q^{2}-1\right) K_{1}+2 K_{1}$ by Lemma 15 , we have the following corollary.

COROLLARY 18. For any integer $n>2$, the graph $\Gamma\left(\mathbb{Z}_{n}[i]\right)$ is not a tree.

6. Colouring of $\Gamma\left(\mathbb{Z}_{n}[i]\right)$. A proper colouring of a graph $G$ is a function that assigns a colour to each vertex such that no two adjacent vertices have the same colour. The graph $G$ is $m$ - colourable if it has a proper colouring with $m$ different colours. The chromatic number of $G$, denoted by $\chi(G)$, is the smallest number of colours necessary to produce a proper colouring. A clique of a graph $G$ is a maximal complete subgraph of $G$. 
THEOREM 19. $\chi\left(\Gamma\left(\mathbb{Z}_{2^{n}}[i]\right)=2^{n}-1\right.$.

Proof. $\Gamma\left(\mathbb{Z}_{2}[i]\right)$ is isomorphic to $K_{1}$ and so $\chi\left(\Gamma\left(\mathbb{Z}_{2^{n}}[i]\right)\right)=2^{1}-1=1$. Now assume that $n>1$. It was proved in [9] that $\mathbb{Z}_{2^{n}}[i]$ is a local ring with maximal ideal $<\overline{1}+$ $i>$, and the properties that $\left|\mathbb{Z}_{2^{n}}[i]\right|=2^{2 n}$ and $<\overline{1}+i>^{2 n-1} \neq\{\overline{0}\}$. Since $(\overline{1}+i)^{2 n-1}=$ $(\overline{1}+i)^{2 n-2}(\overline{1}+i)=(\overline{2} i)^{n-1}(\overline{1}+i) \neq \overline{0}$, it follows by $[4$, Proposition 2.4$]$ that $\Gamma\left(\mathbb{Z}_{2^{n}}[i]\right) \simeq$ $\Gamma\left(\mathbb{Z}_{2^{2 n}}\right)$. Hence it follows by $[7$, Corollary 4.8$]$ that $\chi\left(\Gamma\left(\mathbb{Z}_{2^{n}}[i]\right)=2^{n}-1\right.$.

Lemma 20. Let $R_{1}$ be an integral domain. Then

$\chi\left(\Gamma\left(R_{1} \times R_{2}\right)\right)=\left\{\begin{array}{cl}\chi\left(\Gamma\left(R_{2}\right)\right)+1 & \text { if } Z^{*}\left(R_{2}\right) \neq \phi \\ 2 & \text { if } Z^{*}\left(R_{2}\right)=\phi\end{array}\right.$.

Proof. The vertex set of $\Gamma\left(R_{1} \times R_{2}\right)$ is $\bigcup_{k=1}^{3} A_{k}$, where

$A_{1}=\left\{(x, 0): x \in R_{1}-\{0\}\right\}$,

$A_{2}=\left\{(0, y): y \in R_{2}-\{0\}\right\}$ and

$A_{3}=\left\{(x, z): x \in R_{1}-\{0\}, z \in Z^{*}\left(R_{2}\right)\right\}$.

If $Z^{*}\left(R_{2}\right)=\phi$, then $A_{3}$ is empty and since each of $A_{1}$ and $A_{2}$ is independent in $\Gamma\left(R_{1} \times R_{2}\right)$, we have $\chi\left(\Gamma\left(R_{1} \times R_{2}\right)\right)=2$.

So suppose that $Z^{*}\left(R_{2}\right) \neq \phi$. Since the only non-trivial component of the subgraph of $\Gamma\left(R_{1} \times R_{2}\right)$ induced by $A_{2}$ is isomorphic to $\Gamma\left(R_{2}\right)$, we can colour the vertices in $A_{2}$ by $\chi\left(\Gamma\left(R_{2}\right)\right)$ colours. The vertex $(1,0)$ in $A_{1}$ is adjacent to all vertices in $A_{2}$, so $(1,0)$ must have a new colour. Since $R_{1}$ is an integral domain, $A_{1} \cup A_{3}$ is an independent set of vertices and hence all vertices in $A_{1} \cup A_{3}$ can be coloured by the colour of $(1,0)$. Therefore, $\chi\left(\Gamma\left(R_{1} \times R_{2}\right)\right)=\chi\left(\Gamma\left(R_{2}\right)\right)+1$.

Iterated applications of Lemma 20 lead to the following result.

COROllary 21. If $R$ is a direct product of $n$ integral domains $(n>1)$, then $\chi(\Gamma(R))=n$.

We now calculate the chromatic number for $\Gamma\left(\mathbb{Z}_{n}[i]\right)$ when $n$ is a product of primes congruent to 3 modulo 4 . Similar proofs can be done to find the case when $n$ is a product of primes that are congruent to 1 modulo 4, then the general case can be deduced easily.

THEOREM 22. Let $n=\prod_{k=1}^{r} q_{k}^{m_{k}} \times \prod_{k=r+1}^{t} q_{k}^{m_{k}}, m_{k}>1$ is odd for all $k \leq r$ while $m_{k}$ is non-zero even integer otherwise and let $s=\prod_{k=1}^{t} q_{k}^{2\left\lfloor\frac{m_{k}}{2}\right\rfloor}$. Then

$\chi\left(\Gamma\left(\mathbb{Z}_{n}[i]\right)\right)=s+r-1$.

Proof. Note first that $\mathbb{Z}_{n}[i] \simeq \prod_{k=1}^{t} \mathbb{Z}_{q_{k} m_{k}}[i]$.

Let $S=\left\{\left(\alpha_{k}\right)_{k=1}^{t} \in \prod_{k=1}^{t} \mathbb{Z}_{q_{k} m_{k}}[i]: q_{k}^{\left\lceil\frac{m_{k}}{2}\right\rceil} \mid \alpha_{k}\right.$ for each $\left.k\right\}$. Then elements of $S-$ $\{(\overline{0}, \overline{0}, \overline{0}, \ldots, \overline{0})\}$ form a complete subgraph with $\frac{n^{2}}{\left(\prod_{k=1}^{t} q_{k}^{\left\lceil\frac{m_{k}}{2}\right\rceil}\right)^{2}}-1=s-1$ vertices and hence need $s-1$ different colours. If $v=\left(\beta_{k}\right)_{k=1}^{t} \in Z^{*}\left(\prod_{k=1}^{t} \mathbb{Z}_{q_{k} m_{k}}[i]\right)-S$, then there exists $j$ such that $q_{j}^{\left\lceil\frac{m_{j}}{2}\right\rceil} \nmid \beta_{j}$. Thus $Z^{*}\left(\prod_{k=1}^{t} \mathbb{Z}_{q_{k} m_{k}}[i]\right)-S=A \cup B$, where $A=\bigcup_{j=1}^{t} A_{j}$ with $A_{j}=\left\{\left(\beta_{k}\right)_{k=1}^{t} \in Z^{*}\left(\prod_{k=1}^{t} \mathbb{Z}_{q_{k} m_{k}}[i]\right)-S: q_{j}^{\left\lfloor\frac{m_{j}}{2}\right\rfloor} \nmid \beta_{j}\right\}-\bigcup_{k=1}^{j-1} A_{k}$ and $B=\bigcup_{j=1}^{r} B_{j}$ with $B_{j}=\left\{\left(\beta_{k}\right)_{k=1}^{t} \in Z^{*}\left(\prod_{k=1}^{t} \mathbb{Z}_{q_{k}^{m_{k}}}[i]\right)-S: \beta_{j}=\alpha q_{j}^{\left\lfloor\frac{m_{j}}{2}\right\rfloor}\right.$, where $\alpha$ is a unit in $\mathbb{Z}_{q_{k}^{m_{k}}}[i]$ and $q_{k}^{\left\lfloor\frac{m_{k}}{2}\right\rfloor} \mid \beta_{k}$ for all $\left.k=1,2, \ldots, t\right\}-\bigcup_{k=1}^{j-1} B_{k}$. Elements of $A_{j}$ are independent and 
can be coloured by the colour of $\left(\overline{0}, \overline{0}, \ldots, q_{j}^{\left\lceil\frac{m_{j}}{2}\right\rceil}, \ldots, \overline{0}\right) \in S$. Elements of $B_{j}$ are also independent but are all adjacent to all elements in $S-\{(\overline{0}, \overline{0}, \overline{0}, \ldots, \overline{0})\}$ and so elements of $B_{j}$ can be coloured by a new colour say $c_{j}$. Thus $\Gamma\left(\mathbb{Z}_{n}[i]\right)$ is $(s+r-1)$-colourable and $\chi\left(\mathbb{Z}_{n}[i]\right) \leq s+r-1$. Now $(S-\{(\overline{0}, \overline{0}, \overline{0}, \ldots, \overline{0})\}) \bigcup\left\{v_{1}, v_{2}, \ldots, v_{r}\right\}$ form a clique in $\Gamma\left(\mathbb{Z}_{n}[i]\right)$, where $v_{j}=\left(\beta_{k}\right)_{k=1}^{t}$ with

$$
\beta_{k}=\left\{\begin{array}{l}
q_{j}^{\left\lfloor\frac{m_{j}}{2}\right\rfloor} k=j \\
q_{k}^{\left\lceil\frac{m_{k}}{2}\right\rceil} k \neq j
\end{array} .\right.
$$

Hence $\chi\left(\Gamma\left(\mathbb{Z}_{n}[i]\right)\right)=s+r-1$.

COROllary 23. Let $n>1$. Then

(1) $\chi\left(\Gamma\left(\mathbb{Z}_{n}[i]\right)=m\right.$ if $n=\prod_{k=1}^{m} q_{k}$.

(2) $\chi\left(\Gamma\left(\mathbb{Z}_{q^{n}}[i]\right)=q^{n}-1\right.$ if $n$ is even.

(3) $\chi\left(\Gamma\left(\mathbb{Z}_{q^{n}}[i]\right)=q^{n-1}\right.$ if $n$ is odd.

Note that $\Gamma\left(\mathbb{Z}_{q^{2}}[i]\right)$ is the complete graph $K_{q^{2}-1}$ and so $\chi\left(\Gamma\left(\mathbb{Z}_{q^{2}}[i]\right)=q^{2}-1\right.$.

Since $\mathbb{Z}_{p^{m}}[i] \simeq \mathbb{Z}_{p^{m}} \times \mathbb{Z}_{p^{m}}$, then a similar argument to the proof of Theorem 22 gives the following results:

THEOREM 24. Let $n=\prod_{k=1}^{r} p_{k}^{m_{k}} \times \prod_{k=r+1}^{t} p_{k}^{m_{k}}, m_{k}$ is odd for all $k \leq r$ while $m_{k}$ is non-zero even integer otherwise and let $s=\prod_{k=1}^{t} p_{k}^{2\left\lfloor\frac{m_{k}}{2}\right\rfloor}$. Then

$\chi\left(\Gamma\left(\mathbb{Z}_{n}[i]\right)\right)=s+2 r-1$.

COROllary 25. Let $n>1$. Then

(1) $\chi\left(\Gamma\left(\mathbb{Z}_{n}[i]\right)=2 m\right.$ if $n=\prod_{k=1}^{m} p_{k}$.

(2) $\chi\left(\Gamma\left(\mathbb{Z}_{p^{n}}[i]\right)=p^{n}-1\right.$ if $n$ is even.

(3) $\chi\left(\Gamma\left(\mathbb{Z}_{p^{n}}[i]\right)=p^{n-1}+1\right.$ if $n$ is odd.

Note that $\Gamma\left(\mathbb{Z}_{p}[i]\right)$ is the complete bipartite graph $K_{p-1, p-1}$ and can be coloured by $p^{1-1}+1=2$ different colours.

Combining the work done above, one can conclude the general formula for the chromatic number of $\Gamma\left(\mathbb{Z}_{n}[i]\right)$.

THEOREM 26. Let $n=2^{l} \times\left(\prod_{k=1}^{r} q_{k}^{m_{k}} \times \prod_{k=r+1}^{t} q_{k}^{m_{k}}\right) \times\left(\prod_{k=1}^{z} p_{k}^{n_{k}} \times \prod_{k=z+1}^{c} p_{k}^{n_{k}}\right)$, $m_{k}$ is odd for all $k \leq r$ while $m_{k}$ is even integer otherwise, $n_{k}$ is odd for all $k \leq z$ while $n_{k}$ is even integer otherwise and let $s=2^{l} \times \prod_{k=1}^{t} q_{k}^{2\left\lfloor\frac{m_{k}}{2}\right\rfloor} \times \prod_{k=1}^{c} p_{k}^{2\left\lfloor\frac{m_{k}}{2}\right\rfloor}$. Then

$$
\chi\left(\Gamma\left(\mathbb{Z}_{n}[i]\right)\right)=s+r+2 z-1 .
$$

\section{REFERENCES}

1. D. F. Anderson and P. S. Livingston, The zero-divisor graph of a commutative ring, J. Algebra 217(1999), 434-447.

2. F. Buckly and M. Lewinter, A friendly introduction to graph theory (Person Prentice Hall, New Jersy, 2003).

3. P. J. Cameron, Automorphisms of graphs, in: Topics in algebraic graph theory (Beineke L. W. and Wilson R. J., Editors) (Cambridge University Press, Cambridge, UK, 2005), 137-155. 
4. H-J. Chiang-Hsieh and H-J. Wang, Commutative rings with toroidal zero-divisor graphs, Houst. J. Math. 36(1) (2010), 1-31.

5. G. Dersden and W. M. Dymàček, Finding factors of factor rings over the Gaussian integers, Amer. Math. Monthly 112(7) (2005), 602-611.

6. M. Doob, Spectral graph theory, in: Handbook of Graph Theory (Gross J. L. and Yellen J., Editors) (CRC Press LLC, Boca Raton, 2004), 557-573.

7. A. Duane, Proper colorings and p-partite structures of the zero divisor graph, Rose Hulman Undergrad. Math. J. 7(2) (2006), 1-7.

8. E. A. Osba, S. Al-Addasi and N. A. Jaradeh, Zero divisor graph for the ring of Gaussin integers modulo n. Comm. Algebra 36(10) (2008), 3865-3877.

9. E. A. Osba, M. Henriksen, O. Alkam and F. Smith, The maximal regular ideal of some commutative rings. Comment. Math. Univ. Carolinea 47(1) (2006), 1-10.

10. R. Wilson, Introduction to graph theory, 4th edn. (Pearson Prentice Hall, Malaysia, 1996). 